\title{
Travelling wave solutions to a haptotaxis-dominated model of malignant invasion
}

\author{
B P Marchant ${ }^{1}$, J Norbury $^{2}$ and J A Sherratt ${ }^{3}$ \\ ${ }^{1}$ Silsoe Research Institute, Wrest Park, Silsoe MK45 4HS, UK \\ and \\ Centre for Mathematical Biology, Mathematical Institute, 24-29 St Giles', Oxford OX1 3LB, UK \\ ${ }^{2}$ Mathematical Institute, 24-29 St Giles', Oxford OX1 3LB, UK \\ ${ }^{3}$ Department of Mathematics, Heriot-Watt University, Edinburgh EH14 4AS, UK \\ E-mail: ben.marchant@bbsrc.ac.uk
}

Received 23 January 2001, in final form 5 September 2001

Published 22 October 2001

Online at stacks.iop.org/Non/14/1653

Recommended by D Bensimon

\begin{abstract}
A haptotaxis-dominated model of cell invasion is considered for small cell diffusion and fast protease adjustment to the cell-collagen matrix interaction. A simplified limit model has travelling wave cell invasion profiles that are blunt, that is end with a 'shock-like' step, and that evolve stably from initial data that lie to one side of some initial plane. In common with diffusiondominated systems, the travelling wave which evolves from such initial data has the minimum wavespeed permissible in the model. This minimum wavespeed is not, however, determined by the local stability of the steady states in the travelling wave phase plane, but by a novel combination of singular behaviour within the phase plane and hyperbolic shock conditions. It is shown that more accurate models including the detailed fast dynamics of the protease require small amounts of diffusion (of the same order as the fast dynamics timescale) in order to remain stable. However, small diffusion and fast protease adjustment then give physically relevant and interesting solutions that evolve from semicompact initial data and stably invade at speeds well predicted by the simple model.
\end{abstract}

Mathematics Subject Classification: 34E05, 35L65, 70K05, 92C17

\section{Introduction}

Perumpanani et al [9] devised a three-variable model of malignant invasion with the aim of determining a relationship between the speed of invasion of the malignant cells into connective 
tissue and the biological parameters in the model. In a previous study [7] we were able to approximate analytically the invasion speed of solutions to a two-variable limit model of Perumpanani et al's model. These solutions had a novel structure which could be investigated within a highly singular phase plane. In this paper we investigate whether or not these solutions are a valid approximation of solutions to the full model.

The dominant motility mechanism in Perumpanani et al's model is haptotaxis, the directed motion of the malignant cells up the gradient of connective tissue. This gradient is created by proteases, a group of enzymes emitted by the tumour cells which then degrade the connective tissue. Under the assumption that the protease operates on a faster time-scale than the malignant cells or connective tissue the three-variable model may be reduced to a two-variable limit model. In [7] we discovered a novel family of travelling wave solutions to a simplified or reduced model of this malignant invasion. Of particular interest was the slowest member of this family of waves which was seen to evolve from semi-compact initial data (where the initial level of the invading cells falls to zero for $x$ large enough), and was therefore considered to be the most biologically relevant since the invading cells are not present far in front of the invading interface. The invading cells, of concentration $u(x, t)$, have a shock at the front of their wave profile which formed a blunt interface between $u>0$ and $u=0$ (figure 1). By transforming to travelling wave coordinates the nature of these solutions can be studied in a two-dimensional phase plane which exhibits interesting singular behaviour.

The wavespeed of this biologically relevant solution is used as a measure of the tumour's invasiveness. Following non-dimensionalization the model contains only one parameter, namely $\hat{c}$ the scaled concentration of connective tissue in the absence of the tumour. A relationship between this parameter and the wavespeed $a$ can be approximated within the phase plane.

The behaviour of travelling wave solutions for systems where the dominant motility mechanism is diffusion has been well studied [4]. Such systems have been used to model many types of invasive behaviour, examples include the progression of epidemics, the dispersal of insects, chemical concentration waves seen in the Belousov-Zhabotinskii reaction, the influx of healthy cells into wounded tissue and the movement of micro-organisms into a food source. A review of these examples and the techniques used in analysing the corresponding travelling waves is given by Murray [8]. The generic example of a diffusion-dominated process exhibiting travelling waves is the Fisher equation [3]

$$
\frac{\partial u}{\partial t}=u(1-u)+D \frac{\partial^{2} u}{\partial x^{2}}
$$

with positive diffusion coefficient $D$. This was suggested as a deterministic version of a stochastic model for the spatial spread of a favoured gene into a population, where $u(x, t)$ is the proportion of the population expressing the gene at time $t$ and position $x$. Semicompact initial data for this equation are known to evolve to travelling waves of speed $2 \sqrt{D}$ [4]. This wavespeed, the minimum wavespeed of travelling wave solutions of (1), is determined by investigating the local stability of steady states in the corresponding travelling wave phase plane. Semi-compact initial data for our reduced haptotaxis model similarly evolve to the travelling wave with the slowest wavespeed. In this case, however, the minimum wavespeed is determined by novel singular behaviour within the travelling wave phase plane [7].

In this paper we look at the full three-equation system that models the biological behaviour more accurately, rather than the reduced two-equation system, based upon letting a small physical parameter tend to zero, studied in [7]. We discuss whether or not the 'blunt interface' 


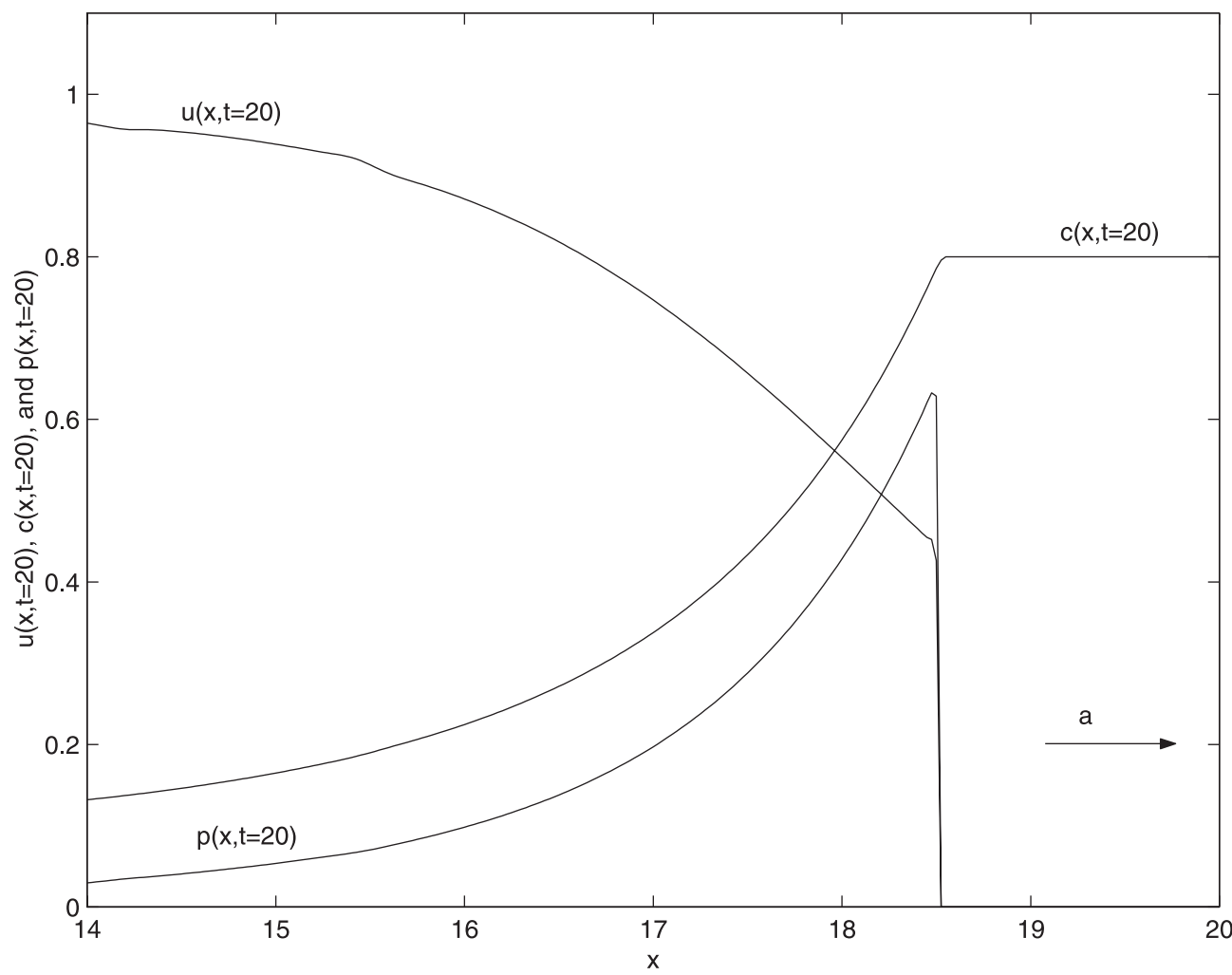

Figure 1. A typical numerical solution of the reduced model of malignant invasion (5) and (6) studied in [7]. This solution evolves from semi-compact initial data. The population of malignant cells $u(x, t)$ at scaled carrying capacity of unity behind the wave, invades at constant speed $a$ into the connective tissue, $c(x, t)$, of density $\hat{c}=0.8$ in front of the wave. The connective tissue is degraded by protease $p(x, t)$ which only exists at the interface of malignant cells and connective tissue. The shape of these wave profiles remains fixed as they move forwards. The shock or 'blunt interface' at the front of the $u$ and $p$ profiles can be clearly seen as well as the discontinuous derivative in the $c$ profile. This solution was obtained using a Kurganov-Tadmor numerical solver [5].

travelling wave solutions also exist for the full model or whether they are merely a mathematical curiosity, resulting from the forcing of trajectories onto a particular surface of the threedimensional travelling wave phase space. Furthermore, we investigate the validity of the approximated relationship between $\hat{c}$ and the invasion speed $a$ derived in [7].

We find that the blunt interface solutions do not exist for the idealized three-equation system without diffusion. Numerical results suggest that semi-compact initial data do not evolve to a stable travelling wave. Instead there is some sort of numerical blow-up near the interface in finite time. Stable travelling wave solutions do exist, however, when a small quantity of diffusion is added to the system. For these solutions the shocks seen in [7] are smoothed within a narrow rescaled inner layer which is the practical interface between the invading $u>0$ and the $u=0$ states. The profiles within this layer contain oscillations, for small diffusion coefficients, with the amplitude of these oscillations increasing as the diffusion coefficient is decreased. A typical example is shown in figure 2. Away from this inner layer the solution profiles are similar to those for the reduced model, and if haptotaxis dominates 


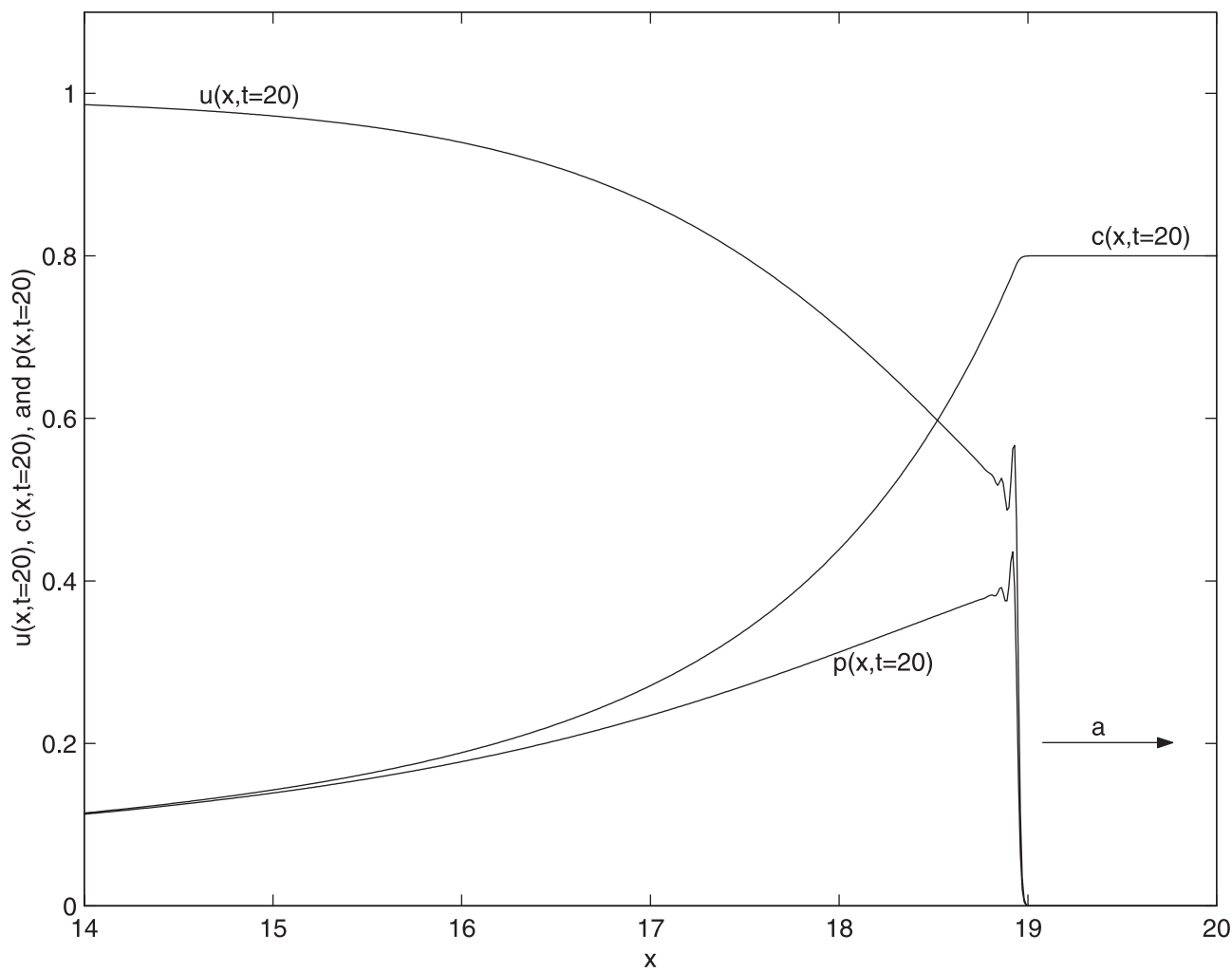

Figure 2. The solution profiles of a numerical solution of Perumpanani et al's full model of malignant invasion including linear diffusion (31)-(33). These profiles have evolved from semicompact initial data. The parameter values in this case are $\hat{c}=0.8, \epsilon=0.01, D_{u}=0.0025$, $D_{c}=D_{p}=0$. The profiles are of a similar form to those for the reduced model shown in figure 1 except the shocks in $u$ and $p$ have been smoothed and oscillations exist at the top of these smoothed shocks. Details of such a solution are contained in section 5.

diffusion the wavespeed of the solution to these equations tends to that of the reduced model. However, as shown in figure 3, if the haptotactic flux is reduced so that diffusion is the dominant motility process then the wavespeed tends to $2 \sqrt{D_{u}}$ (for positive diffusion coefficient $D_{u}$ ), the wavespeed associated with biologically relevant solutions of the Fisher equation (1). Hence the reduced model gives the essential information of the more accurate model. The addition of some diffusion to the system is realistic. It was only omitted from the original model in order to concentrate on the effect of haptotaxis, the directed motion of cells up an insoluble chemical gradient, and to simplify the analysis.

We conclude that the solutions described in [7] are relevant to the study of the larger system with added diffusion in cases where the diffusion is dominated by haptotaxis. The analytical methods described in [7] approximate the behaviour of solutions to a fourth-order PDE (partial differential equation) system by considering a second-order ODE (ordinary differential equation) system combined with the Rankine-Hugoniot jump conditions. This allows an analytical approximation to the wavespeed of the solution to be derived.

Similar methods may be applicable to approximating the wavespeed of travelling wave solutions to other systems where the dominant motility mechanism is an advection process of hyperbolic type, rather than diffusion. 


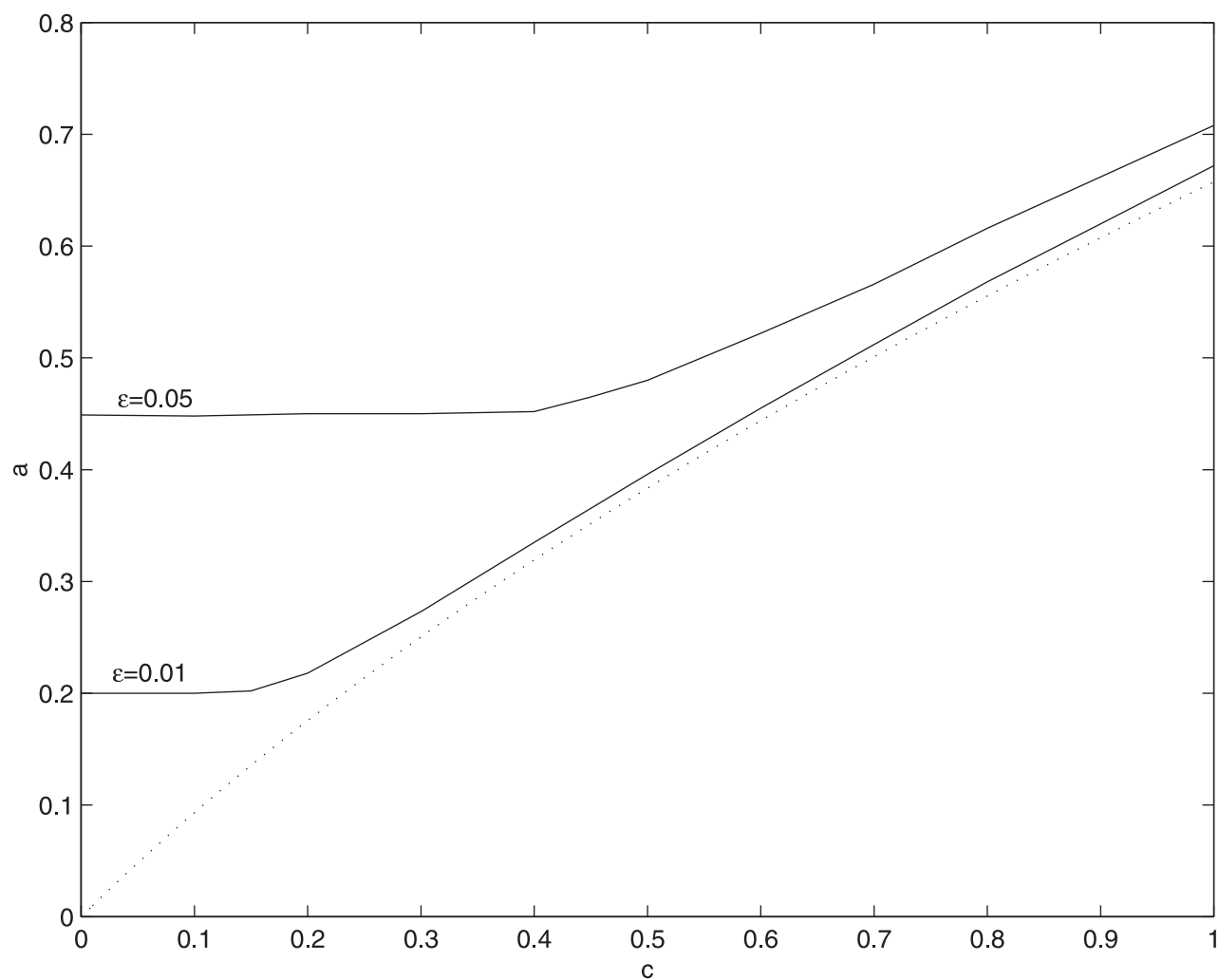

Figure 3. A plot of the minimum wavespeed, $a$, of travelling wave solutions to Perumpanani et al's full model with diffusion (31)-(33), against the steady-state tissue concentration $\hat{c}$. The dotted curve represents the predicted relationship between $a$ and $\hat{c}$ derived from a study of the reduced model (5) and (6). The lower full curve shows the relationship between $a$ and $\hat{c}$ when $\epsilon=D_{u}=0.01, D_{c}=D_{p}=0$. For the other full curve $\epsilon=D_{u}=0.05$. In both cases the curves follow the predicted relationship for large $\hat{c}$. When $\hat{c}$ is small, however, the haptotactic flux is reduced and diffusion is the dominant motility process. Therefore, for small $\hat{c}, a \approx 2 \sqrt{D_{u}}$, as is typical for diffusion driven travelling wave solutions. Details of this relationship are contained in section 5 .

In section 2 we describe the formulation of the model and summarize the properties of the blunt interface solutions derived in [7]. In section 3 we attempt to find similar solutions to the full malignant invasion model (at first without diffusion) but demonstrate that travelling waves with a shock at the front of the invading wave profile do not then exist. The numerical evidence detailed in section 4 suggests that, when an accurate numerical solver with minimal numerical diffusion is used, semi-compact initial data applied to this model do not evolve to a stable travelling wave solution. Stable travelling wave solutions may be seen, however, if a first-order accurate numerical scheme, which adds numerical diffusion to the equations, is used. Therefore, in section 5 we investigate analytically why the addition of small amounts of diffusion may lead to these stable solutions, establishing the existence of a stable smooth inner transition layer where the 'shock-like' blunt profile of the simple model occurs, and that the simple model solution is a good approximation when only a small amount of diffusion is present. 


\section{Travelling shock waves}

In [7] we found a novel family of travelling wave solutions to an approximation of Perumpanani et al's malignant invasion model formulated in [9]. The original model depends upon one space direction $x$ and time $t$ and has three generic variables, namely $u(x, t)$ the concentration of invasive cells, $c(x, t)$ the concentration of connective tissue (which is made of extracellular matrix elements) and $p(x, t)$ the concentration of protease. All concentrations are measured per unit volume at position $x$ and time $t$; the model studies the averaged behaviour of cells which vary in the direction of invasion only, and therefore the model ignores variations in the plane perpendicular to the axis of invasion.

The major motility mechanism governing the behaviour of the malignant cells is haptotaxis up the gradient of connective tissue. This is represented by an invasive flux proportional to $u \partial c / \partial x$ (and thus an invasive velocity proportional to $\partial c / \partial x$ ). Perumpanani et al [9] also include logistic proliferation of the malignant cells via the non-dimensionalized term $u(1-u)$. With no connective tissue gradient present (that is, $\partial c / \partial x \equiv 0$ ), the malignant cells will grow from small densities to the non-dimensionalized steady-state carrying capacity scaled to be 1 . This capacity is a result of inhibition of the division of cells by their neighbours, via mechanisms generically referred to as contact inhibition.

Upon contact with connective tissue the invasive cells produce protease of density $p(x, t)$ at a rate proportional to $u c$. Protease dissolves the connective tissue at a rate proportional to $c p$. The protease itself undergoes natural decay proportional to its own concentration.

Following non-dimensionalization which removes all but one of the parameter values, this model is written as

$$
\begin{aligned}
\frac{\partial u}{\partial t} & =u(1-u)-\frac{\partial}{\partial x}\left(u \frac{\partial c}{\partial x}\right) \\
\frac{\partial c}{\partial t} & =-p c \\
\frac{\partial p}{\partial t} & =\frac{1}{\epsilon}(u c-p)
\end{aligned}
$$

where $\epsilon$ is a positive constant representing the relative timescale of the protease dynamics to that of the cell growth dynamics. Here $t$ is scaled so that $u$ grows on the $\mathrm{O}(1)$ timescale to the $u$ scaled carrying capacity of unity, $x$ is scaled so that the rate of haptotaxis is of the same order, $p$ is scaled so that $c$ dissolves on the same timescale and, finally, $c$ is scaled so that $p$ and $u c$ are of the same order in equation (4). This leaves the $p$ timescale relatively much faster, so that $\epsilon$ is small.

The situation being considered is an invasion of malignant cells (from a large negative $x$ direction) at their carrying capacity density into connective tissue of some positive concentration $\hat{c}$ (at a large positive $x$ direction). Thus $\hat{c}$ is a second parameter of the system. This model PDE system is solved on the infinite- $x$ domain, with boundary behaviour $u=1, c=0, p=0$ at $x=-\infty$ and $u=0, c=\hat{c}, p=0$ at $x=\infty$.

In [10] Perumpanani et al approximated (2)-(4) by a two-equation system. Exploiting the small parameter $\epsilon$, they approximated the protease dynamics (4) by $p=u c+\mathcal{O}(\epsilon)$; this is appropriate provided that $\partial p / \partial t$ is bounded. By setting $p=u c$ the three-variable problem could be reduced to the two-variable problem

$$
\begin{aligned}
\frac{\partial u}{\partial t} & =u(1-u)-\frac{\partial}{\partial x}\left(u \frac{\partial c}{\partial x}\right) \\
\frac{\partial c}{\partial t} & =-u c^{2} .
\end{aligned}
$$


Here the only parameter is $\hat{c}$.

In [7] we described three families of travelling wave solution to this reduced model. We now concentrate upon the particular solution which appears to be most biologically relevant since it evolves from the most general initial condition profiles. We refer to this solution as the blunt interface solution since there is a blunt interface at the front of the $u(x, t)$ profile (see figure 1).

We look for constant shape travelling waves by setting

$$
u(x, t) \equiv U(z) \quad c(x, t) \equiv C(z) \quad z \equiv x-a t .
$$

The PDE system (5) and (6) is then reduced to the following third-order ODE system:

$$
\begin{aligned}
& -a \frac{\mathrm{d} U}{\mathrm{~d} z}=U(1-U)-\frac{\mathrm{d}}{\mathrm{d} z}\left(U \frac{\mathrm{d} C}{\mathrm{~d} z}\right) \\
& a \frac{\mathrm{d} C}{\mathrm{~d} z}=U C^{2} .
\end{aligned}
$$

Here $U \rightarrow 1, C \rightarrow 0$ as $z \rightarrow-\infty$ and $U \rightarrow 0, C \rightarrow \hat{c}$ as $z \rightarrow \infty$, with $\hat{c}$ a positive parameter. The wavespeed $a$ is unknown and determined by the $C \rightarrow \hat{c}$ boundary condition.

The key to our method of analysis is the fact that there are no $z$ derivative terms on the right-hand side of (9). This allows us to substitute (9) for $\mathrm{d} C / \mathrm{d} z$ in (8), thus reducing the system to the second-order non-standard form

$$
\begin{aligned}
& \frac{\mathrm{d} U}{\mathrm{~d} z}\left(-a+\frac{2 U C^{2}}{a}\right)=U(1-U)-2 U^{3} C^{3} a^{-2} \\
& \frac{\mathrm{d} C}{\mathrm{~d} z}=\frac{U C^{2}}{a} .
\end{aligned}
$$

Travelling wave solutions are then represented by $(U, C)$ phase plane trajectories which leave the malignant steady state $U=1, C=0$ and connect to the healthy steady state $U=0, C=\hat{c}$. Local stability analysis of these steady states reveals that the malignant one possesses an unstable manifold, and the healthy one a stable manifold, as required for a connection of this type to exist.

The interesting point to note about (10) and (11) is that when $2 U C^{2}=a^{2},(10)$ becomes singular. We refer to this curve as the singular barrier, ' $\mathrm{S}$ '. Phase plane trajectories have no meaning on the singular barrier and cannot normally cross this curve. The one exception to this is when the $U$ nullcline, ' $\mathrm{N}$ ',

$$
U(1-U)-\frac{2 U^{3} C^{3}}{a^{2}}=0
$$

intersects the singular barrier, since at this point the derivatives may still be finite because of the zero/zero indeterminate form. We call this point the 'hole in the wall'. The hole in the wall was first identified by Pettet et al [11] in a model of angiogenesis. In the following discussion we fix $a>0$ and regard $\hat{c}$ as the resulting parameter to be determined. We show in [7] that $a$ and $\hat{c}$ vary monotonically (see figure 3 ).

The trajectory corresponding to the blunt interface travelling wave solution which we concentrate upon does, in fact, cross the singular barrier at the hole in the wall. Its progress towards a healthy steady state, $U=0, C=\hat{c}$ then appears to be blocked by the singular barrier. The connection between the steady states is completed via a hyperbolic discontinuity [2] which allows the trajectory to jump over the singular barrier. In [7] we demonstrate that the PDE system (5) and (6) is strictly hyperbolic and hence solutions to it may contain discontinuities. Combining the Rankine-Hugoniot jump conditions [2] with the assumption of travelling wave 

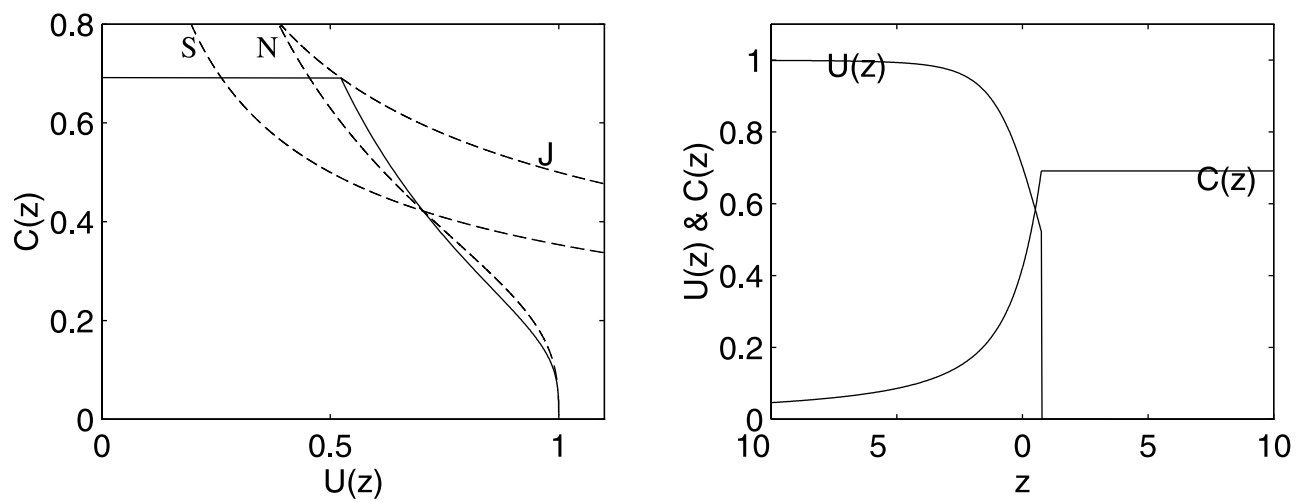

Figure 4. A phase plane plot and a solution profile for the 'blunt interface' travelling wave solution of (5) and (6). Here $a=0.5$. In the phase plane a travelling wave solution is represented by a connection between the steady state at $z=-\infty(U=1, C=0)$, and the steady state at $z=\infty$ $(U=0, C=\hat{c})$. This connecting trajectory crosses the singular barrier ' $\mathrm{S}$ ' at the hole in the wall (the point at which the singular barrier intersects the $U$ nullcline, ' $\mathrm{N}$ '). The singular barrier then appears to block the trajectory from connecting smoothly to the $C$-axis. A hyperbolic jump may, however, take the trajectory from the curve ' $\mathrm{J}$ ' $\left(U=a^{2} / C^{2}\right)$, to the $C$-axis. In the solution profile, the blunt interface in the $U$ profile can be clearly seen as can the corner in the $C$ profile.

solutions reveals that a phase plane trajectory may jump from the ' $\mathrm{J}$ ' curve $U C^{2}=a^{2}$ to the set of healthy steady states on the $C$-axis. We can show that for all values of $a$, there exists one trajectory which leaves $U=1, C=0$, crosses the singular barrier at the hole in the wall and then goes on to hit the ' $\mathrm{J}$ ' curve $U C^{2}=a^{2}$ from where it is able to jump to a healthy steady state on the $C$-axis. Such a steady-state connection and the corresponding travelling wave profile are shown in figure 4.

This particular solution has many desirable properties. The discontinuity satisfies the Lax entropy condition and is therefore stable to added diffusion. Since the solution can be studied in the phase plane it is simple to estimate a relationship between the parameter $\hat{c}$ and the travelling wavespeed $a$ (by approximating the value of $C=\hat{c}$ where the trajectory crosses the ' $\mathrm{J}$ ' curve $U C^{2}=a^{2}$ ). In [7] we show that a leading-order approximation of this relationship for small $\hat{c}$ is

$$
a=\frac{-1+(1+\hat{c}(6-2 \sqrt{2}))^{1 / 2}}{3-\sqrt{2}} .
$$

Numerical investigations show that the blunt interface solution is stable and evolves from biologically relevant initial data for the PDEs (5) and (6) where $u(x, t=0)$ has semi-compact support (that is, $u(x, 0)$ vanishes for $x$ greater than some $\left.x_{0}\right)$. Furthermore, the leading-order approximation of its wavespeed is accurate for $0<\hat{c}<3$.

In this paper we consider the three-equation system (2)-(4) and discuss whether or not biologically relevant solutions to it can be approximated by the travelling shock wave described above. In making the $p=u c+\mathcal{O}(\epsilon)$ approximation we assume that $\partial p / \partial t$ is bounded. We have described how the solution contains a jump in $u$ and hence in $p$ (since $p=u c$ ) so this boundedness assumption is not valid throughout the solution. Therefore, the discontinuous solution does not form the leading term of a regular approximation in $\epsilon$, and instead some sort of rescaling of the space variable is required close to the shock. As $\epsilon \rightarrow 0$ the solutions in the inner layer should tend to the shocks found in the reduced model (5) and (6). The new inner solution should match with the non-jump parts of the blunt interface solution. We 
now assume that the blunt interface solution forms a valid leading-order solution of the full model (2)-(4) and henceforth refer to it as $u_{0}(x, t), c_{0}(x, t), p_{0}(x, t) \equiv u_{0} c_{0}$. We will show that this assumption is only correct if diffusion is added to the model. In the next section we investigate whether or not the inner layer may still contain discontinuities.

\section{Looking for travelling shock wave solutions to the full equations}

In section 2 we discussed how semi-compact initial conditions to (5) and (6) evolve to novel travelling wave solutions which have a discontinuity at the front of the advancing wave of invading cells. In this section we look at the three-equation model (2)-(4) and discuss whether or not it possesses similar solutions and whether or not the same methods of analysis are directly applicable.

Transforming the full PDEs (2)-(4) into travelling wave coordinates $(z \equiv x-a t)$ yields, after manipulation to reduce the fourth-order system to third order similar to that reducing equations (8) and (9) to (10) and (11),

$$
\begin{aligned}
& \frac{\mathrm{d} U}{\mathrm{~d} z}\left(a^{3}-a C P\right)=-a^{2} U(1-U)+U C P^{2}+\frac{U C}{\epsilon}(P-U C) \\
& \frac{\mathrm{d} C}{\mathrm{~d} z}=\frac{C P}{a} \\
& \frac{\mathrm{d} P}{\mathrm{~d} z}=\frac{1}{\epsilon a}(P-U C) .
\end{aligned}
$$

Note that if $\mathrm{d} P / \mathrm{d} z$ is bounded then $P=U C+\mathcal{O}(\epsilon)$, and so $(P-U C) / \epsilon$ may be eliminated from (14) by using $(P-U C) / \epsilon=a \mathrm{~d} P / \mathrm{d} z=a \mathrm{~d}(U C) / \mathrm{d} z+\mathcal{O}(\epsilon)$, giving the reduced system (10) and (11) to leading order in $\epsilon \rightarrow 0$. The boundary conditions are $(U, C, P) \rightarrow(1,0,0)$ as $z \rightarrow-\infty$ (the malignant state) and $(U, C, P) \rightarrow(0, \hat{c}, 0)$ as $z \rightarrow \infty$ (the healthy steady state). Local stability analysis suggests that these steady states are suitable boundary conditions. For a given $a, \epsilon$ we now seek an orbit from $(1,0,0)$ to $U=P=0$ which determines $\hat{c}$.

In common with the $\left(U_{0}, C_{0}\right)$ phase plane described in the last section, the $(U, C, P)$ phase space possesses a singular barrier. Equation (14) becomes singular, for any $\epsilon>0$, when

$$
a^{2}-C P=0
$$

The singular barrier of the reduced model (where $P_{0}=U_{0} C_{0}$ ) was $2 U_{0} C_{0}^{2}=a^{2}$, thus the full equations have a different singular barrier. In fact, in the case $\epsilon=0$, equation (17) corresponds to the ' $\mathrm{J}$ ' curve $U_{0} C_{0}^{2}=a^{2}$, which is the curve from which $U_{0}$ profiles of the reduced model were seen to jump down to zero. We are now working in three-dimensional phase space and the singular barrier is a two-dimensional surface. The only points on the barrier which trajectories may approach with a finite gradient are holes in the wall, where the singular barrier intersects the $U$ nullcline. The $U$ nullcline now consists of the plane and surface (respectively)

$$
\begin{aligned}
& U=0 \\
& -a^{2}(1-U)+C P^{2}+\frac{C}{\epsilon}(P-U C)=0 .
\end{aligned}
$$

Thus there are two curves of holes in the wall, where these surfaces intersect the singular barrier $C P=a^{2}$; however, we do not expect physically relevant trajectories to cross $U=0, C>0, P=a^{2} / C$.

The change in the equation of the singular barrier means that if we transform the smooth part of our blunt solution of the reduced model to this three-dimensional phase space, the 
trajectory does not cross the singular barrier of the full equations; rather, the smooth part of the solution ends on the singular barrier. Thus our solution for $U_{0}(z), C_{0}(z)$ and $P_{0}(z)$ could form a valid leading-order outer solution for the full model, which takes the trajectory from the malignant steady state $U=1, C=0, P=0$ to the surface $C P=a^{2}$. The question we now ask is whether the connection to one of the family of healthy steady states on the $C$-axis may be completed. Note that the jump values for $U_{0}, C_{0}, P_{0}=U_{0} C_{0}$ are given by $U_{0} C_{0}^{2}=a^{2}$, so that this outer solution appears to jump just as it meets the singular barrier.

First we consider the possible existence of shocks which would complete this connection in a similar manner to our solution to the reduced model. The theory used here is described in [2]. Equations (2)-(4) may be written in conservation form

$$
\frac{\partial}{\partial t}\left(\begin{array}{c}
u \\
v \\
c \\
\epsilon p
\end{array}\right)+\frac{\partial}{\partial x}\left(\begin{array}{c}
u v \\
c p \\
0 \\
0
\end{array}\right)=\left(\begin{array}{c}
u-u^{2} \\
0 \\
-c p \\
u c-p
\end{array}\right)
$$

where $v(x, t) \equiv c_{x}$.

This conservation law has characteristic gradients $\lambda_{1}=\lambda_{2}=\lambda_{3}=0 \leqslant \lambda_{4}=v$. Therefore, the system is weakly hyperbolic, and the $\lambda_{1}, \lambda_{2}$ and $\lambda_{3}$ fields are linearly degenerate. The right eigenvector corresponding to $\lambda_{4}$ is $r_{4}=(1,0,0,0)$ and therefore this field is also linearly degenerate (since $\lambda_{4}^{\prime}(\underline{U}) \cdot r_{4}(\underline{U})=0$ ) and any discontinuities in solutions to this model are contact discontinuities which propagate at speed $\lambda_{i}$.

For a hyperbolic system of the form, for $\underline{U}=(u, v, c, \epsilon p)$,

$$
\underline{U}_{t}+\underline{A}_{x}=\underline{B}
$$

where $\underline{A}, \underline{B}$ are functions of $\underline{U}, x$ and $t$, the Rankine-Hugoniot jump condition in the $k$ th field [2] is

$$
[\underline{U}] s_{k}=[\underline{A}]
$$

here $[\underline{U}]$ denotes the jump in $\underline{U},[\underline{A}]$ the jump in $\underline{A}$ and $s_{k}$ the shock speed.

We ignore the stationary discontinuities in the $\lambda_{1}, \lambda_{2}$ and $\lambda_{3}$ fields as being inconsistent with travelling waves, and look for contact discontinuities in the $\lambda_{4}$ field. If we assume that the solutions are travelling waves moving forwards in space at speed $a$ (i.e. $s_{k}=a$ ) then the jump conditions become

$$
\begin{aligned}
& {[U] a=[U V]} \\
& {[V] a=[C P]} \\
& {[C] a=0} \\
& {[\epsilon P] a=0 .}
\end{aligned}
$$

This implies that for $a, \epsilon \neq 0$

$$
[P]=[C]=[V]=0
$$

and a non-zero jump in $U$ only exists if $V=a$. Therefore, unlike the $\epsilon=0$ case, the $P$ profile must be continuous and the shock in the $p_{0}$ outer solution must somehow be smoothed in a rescaled inner layer.

We now look at the jumps which do exist in solutions of the full equations (2)(4). Combining the jump conditions (23)-(26) with (15) we see that at the discontinuity, $V=\mathrm{d} C / \mathrm{d} z=C P / a$ and $V=a$. Therefore, $C P=a^{2}$ at the jumps of travelling wave 
solutions to the full equations (2)-(4) which travel at speed $a$, and so jumps may only occur at the singular barrier defined by (17). The jump conditions do not determine the length of the jump in $U$, but since the singular barrier may only be approached with a finite gradient at a hole in the wall, the jump must be between the two curves of holes in the wall. Thus one end point will have $U=0$ and the other $-a^{2}(1-U)+C P^{2}+C(P-U C) / \epsilon=0$. In fact, once the trajectory hits the hole in the wall curve with $U>0$, a jump in $U$ only would force the curve to 'slide' down the singular surface $C P=a^{2}$ to the $U=0$ plane with $C, P$ and $V$ remaining constant.

Such a jump in $U$ is consistent with the travelling wave solutions with an interface between $U>0$ and $U=0$ seen with the reduced model. But we also need to consider the continuous behaviour of the $P(z)$ profile. The basic solution we are considering is an outer solution trajectory leaving $U_{0}=1, C_{0}=0, P_{0}=0$ and connecting to a hole in the wall of the full system for $U>0$. From this hole in the wall, $U(z)$ can jump down to zero as required to complete a travelling shock wave. The $P$ profile is continuous, however, so after the jump in $U(z)$ occurs, $P(z)>0$, and according to (16) will continue to increase. Therefore, the trajectory will fail to connect to the $U=0, C=\hat{c}, P=0$ steady state. Even if $U$ did not jump all the way down to zero, equation (16) shows that for $\epsilon$ small, $P$ would have to increase significantly more rapidly than $C$, and then (14) shows that $\mathrm{d} U / \mathrm{d} z<0$ so that $U$ would continue to decrease while $P$ would continue to increase. Again no connection to $(0, \hat{c}, 0)$ is possible.

Therefore, there does not exist a travelling wave solution to the full equations which is of the same form as the blunt interface solution of the reduced model. This is because the jump is forced to occur parallel to, and along, the singular surface. In the next section we look at numerical solutions to the full equations in order to ascertain what form of solutions evolve stably from semi-compact initial data.

\section{Numerical solutions of the full equations}

We solve equations (2)-(4) numerically using a Kurganov-Tadmor [5] scheme. The KurganovTadmor scheme is a second-order accurate solver for hyperbolic equations. Other second-order accurate methods such as Godunov's method [6] require the solution of the equations along the system's characteristics. This can be awkward to implement for nonlinear systems such as ours. This is not the case for the Kurganov-Tadmor scheme which simply requires derivatives within the system to be calculated componentwise.

The initial conditions used are of the form

$$
\begin{aligned}
& u(x, t=0)= \begin{cases}1 & \text { if } \quad x<x_{1} \\
\frac{1}{2} \cos \left(\frac{\pi\left(x-x_{1}\right)}{x_{2}-x_{1}}\right)+\frac{1}{2} & \text { if } \quad x_{1} \leqslant x \leqslant x_{2} \\
0 & \text { if } x>x_{2}\end{cases} \\
& c(x, t=0)=\hat{c}(1-u(x, t=0)) \\
& p(x, t=0)=0 .
\end{aligned}
$$

This is the form of initial data which were seen to evolve to the invading profile with a blunt interface between $u$ positive and $u=0$ for the reduced model (5) and (6). When these initial data were given to the full equation system, however, no travelling wave solutions evolved and numerical instabilities appeared. Interestingly travelling wave solutions did appear when the Lax-Friedrichs scheme [6] was used to solve these equations. Typical examples of these solutions are shown in figure 5 . The $u(x, t)$ profile is no longer monotonic and some oscillatory 

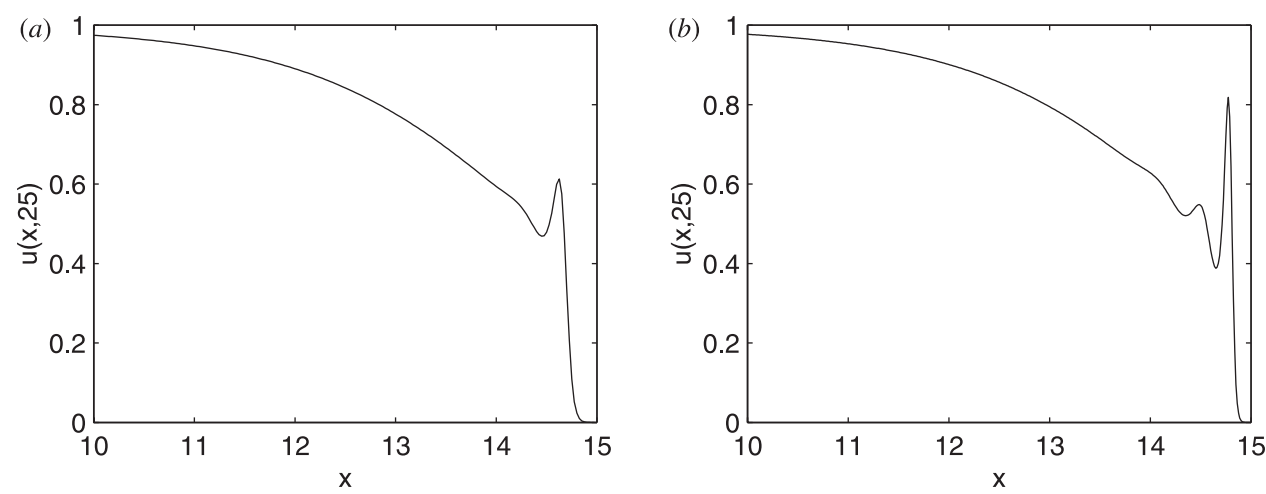

Figure 5. The $u(x, 25)$ profile from Lax-Friedrichs numerical solutions of (2)-(4) evolving from semi-compact $u(x, t=0)$. Here $\hat{c}=1.0$ and $\epsilon=0.1$. (a) $\Delta x=0.025 ;(b) \Delta x=0.0125$. This decrease in the mesh size (and hence decrease in the amount of numerical diffusion) causes the amplitude of the oscillations at the front of the wave to increase.

behaviour has appeared at the front of the $u(x, t)$ profile. The amplitude of these oscillations increases as the steps in the mesh on which the equations are solved, $\Delta x$ and $\Delta t$, are reduced.

The Lax-Friedrichs scheme is only first-order accurate and therefore effectively solves the equations with small diffusion terms added. The size of these diffusion terms decreases as the mesh is refined. It therefore appears that this small amount of diffusion in some way stabilizes the solutions. In the next section we consider the effect of adding a small amount of diffusion to Perumpanani et al's [9] model.

\section{Adding diffusion to the model}

Our numerical evidence suggests that stable travelling wave solutions of (2)-(4) may only evolve from semi-compact $u(x, t=0)$ initial data if a small amount of diffusion is added to the model. This is biologically realistic, since (2)-(4) is merely an approximation to the invasive situation in which diffusion has been neglected. We assume that this diffusion is linear, so we now consider the system

$$
\begin{aligned}
\frac{\partial u}{\partial t} & =u(1-u)-\frac{\partial}{\partial x}\left(u \frac{\partial c}{\partial x}\right)+D_{u} \frac{\partial^{2} u}{\partial x^{2}} \\
\frac{\partial c}{\partial t} & =-p c+D_{c} \frac{\partial^{2} c}{\partial x^{2}} \\
\frac{\partial p}{\partial t} & =\frac{1}{\epsilon}(u c-p)+D_{p} \frac{\partial^{2} p}{\partial x^{2}}
\end{aligned}
$$

where $D_{u}, D_{c}$ and $D_{p}$ are positive diffusion coefficients.

In this section we investigate a leading-order asymptotic approximation to (31)-(33) when a small amount of diffusion of the invading cells is included in the system. We assume that $D_{u}=\mathcal{O}(\epsilon)$ and $D_{c}=D_{p}=0$. This is realistic for some invasive situations. The connective tissue is a large immobile matrix so it is reasonable to assume that $D_{c}=0$. Tsuboi and Rifkin [12] stated that UPA, a protease produced by HT1080 cells, is bound to the extracellular matrix and therefore does not diffuse so we take $D_{p}=0$. In fact, we have found similar results when $D_{p}$ is non-zero. Aznavoorian et al [1] found that HT1080 melanoma cells had 
a haptotactic response more than 50 times greater than the random response, giving some justification for a small value of $D_{u}$.

Our blunt interface solution to the reduced model (5) and (6) forms the outer solution $u_{0}, c_{0}, p_{0}$ for this problem. In the region where $\partial p_{0} / \partial t$ is unbounded a rescaled inner solution $\tilde{u}_{0}, \tilde{c}_{0}, \tilde{p}_{0}$ is required. Each end of this inner solution should match with the values of the outer solution on either side of the shock, where we just take $U=0, C=\hat{c_{0}}, P=0$ for the state of the outer solution after the shock.

If we introduce the $\mathcal{O}(1)$ constant $d_{u}=D_{u} / \epsilon$, the travelling wave equations for the system (31)-(33) become (with $D_{c}=D_{p}=0$ )

$$
\begin{aligned}
& \left(\frac{C P}{a}-a\right) \frac{\mathrm{d} U}{\mathrm{~d} z}=U(1-U)-\frac{U C}{\epsilon a^{2}}(P-U C)+\frac{U C P^{2}}{a^{2}}-\epsilon d_{u} \frac{\mathrm{d}^{2} U}{\mathrm{~d} z^{2}} \\
& \frac{\mathrm{d} C}{\mathrm{~d} z}=\frac{C P}{a} \\
& \frac{\mathrm{d} P}{\mathrm{~d} z}=\frac{1}{\epsilon a}(P-U C) .
\end{aligned}
$$

Here we have eliminated $\mathrm{d} C / \mathrm{d} z$ (using (35)) from equation (34), and have eliminated $\mathrm{d} P / \mathrm{d} z$ (using (36)) so that (34) becomes the same as (14) when $d_{u}=0$.

In regions where all the derivatives are bounded (34)-(36) may be approximated to leading order in $\epsilon$ by (10) and (11), the travelling wave equations studied in [7]. Thus the introduction of diffusion has no effect upon the leading-order outer solution.

When $C P-a^{2}=\mathcal{O}(\epsilon)$ (i.e. close to the shock in the outer solution) however, $\mathrm{d} U / \mathrm{d} z=\mathcal{O}(1 / \epsilon)$ and a rescaling of the $z$ variable is required. Note that if $a^{2}=\mathrm{O}(1)$ then the outer solution approximation is valid at the reduced model (5) and (6) singular barrier (that is $U C^{2}=C P=a^{2} / 2$ ). The approximation is not valid as the solution approaches the full model singular barrier $C P=a^{2}$. In this inner region we introduce $\zeta$ such that $z-z_{0}=\epsilon \zeta$, where $z \rightarrow z_{0}$ as we approach the singular barrier. The leading-order inner equations are then written as

$$
\begin{aligned}
& \left(a^{3}-a \tilde{C}_{0} \tilde{P}_{0}\right) \frac{\mathrm{d} \tilde{U}_{0}}{\mathrm{~d} \zeta}=\tilde{U}_{0} \tilde{C}_{0}\left(\tilde{P}_{0}-\tilde{U}_{0} \tilde{C}_{0}\right)+a^{2} d_{u} \frac{\mathrm{d}^{2} \tilde{U}_{0}}{\mathrm{~d} \zeta^{2}} \\
& \frac{\mathrm{d} \tilde{C}_{0}}{\mathrm{~d} \zeta}=0 \\
& \frac{\mathrm{d} \tilde{P}_{0}}{\mathrm{~d} \zeta}=\frac{1}{a}\left(\tilde{P}_{0}-\tilde{U}_{0} \tilde{C}_{0}\right) .
\end{aligned}
$$

From (38) we see that $\tilde{C}_{0}$ is a constant. The inner layer equations may be rewritten as a first-order system by introducing the new variable $\tilde{W}_{0}:=\mathrm{d} \tilde{U}_{0} / \mathrm{d} \zeta$, so that we have

$$
\begin{aligned}
\frac{\mathrm{d} \tilde{W}_{0}}{\mathrm{~d} \zeta} & =\frac{\tilde{U}_{0} \tilde{C}_{0}}{a^{2} d_{u}}\left(\tilde{P}_{0}-\tilde{U}_{0} \tilde{C}_{0}\right)+\frac{\tilde{W}_{0}\left(\tilde{C}_{0} \tilde{P}_{0}-a^{2}\right)}{a d_{u}} \\
\frac{\mathrm{d} \tilde{U}_{0}}{\mathrm{~d} \zeta} & =\tilde{W}_{0} \\
\frac{\mathrm{d} \tilde{P}_{0}}{\mathrm{~d} \zeta} & =\frac{1}{a}\left(\tilde{P}_{0}-\tilde{U}_{0} \tilde{C}_{0}\right) .
\end{aligned}
$$

Writing (40) in the form

$$
a d_{u} \frac{\mathrm{d} \tilde{W}_{0}}{\mathrm{~d} \zeta}=\tilde{U}_{0} \tilde{C}_{0} \frac{\mathrm{d} \tilde{P}_{0}}{\mathrm{~d} \zeta}+\tilde{C}_{0} \tilde{P}_{0} \frac{\mathrm{d} \tilde{U}_{0}}{\mathrm{~d} \zeta}-a^{2} \frac{\mathrm{d} \tilde{U}_{0}}{\mathrm{~d} \zeta}
$$


and using $\tilde{C}_{0}$ as a constant, we can integrate (40) to find that

$$
\tilde{W}_{0}=\frac{\mathrm{d} \tilde{U}_{0}}{\mathrm{~d} \zeta}=\frac{\tilde{U}_{0} \tilde{C}_{0} \tilde{P}_{0}}{a d_{u}}-\frac{a \tilde{U}_{0}}{d_{u}}+A
$$

for some arbitrary constant $A$. Thus we have reduced the study of the system in the inner layer to the two equations (42) and (44).

We require that $\tilde{U}_{0}, \tilde{C}_{0}, \tilde{P}_{0}$ as $\zeta \rightarrow \pm \infty$ match with the values of $U_{0}, C_{0}, P_{0}$ on either side of the outer solution shock. Thus we require that $\tilde{C}_{0}=\hat{c}_{0}$ throughout the inner layer, and that $\tilde{W}_{0}=0$ (that is, $\mathrm{d} U_{0} / \mathrm{d} z$ is bounded), $\tilde{U}_{0} \hat{c}_{0}^{2}=a^{2}$ and $\tilde{P}_{0}=\tilde{U}_{0} \hat{c}_{0}$ as $\zeta \rightarrow-\infty$, and $\tilde{W}_{0}=\tilde{U}_{0}=\tilde{P}_{0}=0$ as $\zeta \rightarrow \infty$. Both of these points are steady states in the $\left(\tilde{U}_{0}, \tilde{P}_{0}\right)$ phase plane for equations (42)-(44) when $A=0$. Note that the conditions as $\zeta \rightarrow-\infty$ imply that $A=0$ in equation (44). A phase plane connection between these points will be possible if the steady state at $\zeta=-\infty\left(\tilde{U}_{0}=a^{2} / \hat{c}_{0}^{2}, \tilde{P}_{0}=a^{2} / \hat{c}_{0}\right)$ has a non-trivial unstable manifold and the steady state at $\zeta=\infty\left(\tilde{U}_{0}=0, \tilde{P}_{0}=0\right)$ has a non-trivial stable manifold.

The Jacobian of (42) and (44) is

$$
J_{\left(\tilde{U}_{0}, \tilde{P}_{0}\right)}=\left(\begin{array}{cc}
\frac{\hat{c}_{0} \tilde{P}_{0}-a^{2}}{a d_{u}} & \frac{\tilde{U}_{0} \hat{c}_{0}}{a d_{u}} \\
-\frac{\hat{c}_{0}}{a} & \frac{1}{a}
\end{array}\right) .
$$

At the $\zeta=\infty$ steady state this becomes

$$
J_{\left(\tilde{U}_{0}, \tilde{P}_{0}\right)}=\left(\begin{array}{cc}
\frac{-a}{d_{u}} & 0 \\
-\frac{\hat{c}_{0}}{a} & \frac{1}{a}
\end{array}\right) .
$$

Thus this steady state is a saddle point with eigenvalues $\lambda=1 / a$ and $-a / d_{u}$. The trajectory may approach the healthy steady state along the stable eigenvector $\left(1, \hat{c}_{0} d_{u} /\left(d_{u}+a^{2}\right)\right)$. Therefore, as $\zeta \rightarrow \infty$

$$
\begin{aligned}
& \tilde{U}_{0}=B \exp \left(-\frac{a}{d_{u}} \zeta\right) \\
& \tilde{C}_{0}=\hat{c}_{0} \\
& \tilde{P}_{0}=B \frac{\hat{c}_{0} d_{u}}{d_{u}+a^{2}} \exp \left(-\frac{a}{d_{u}} \zeta\right)
\end{aligned}
$$

where $B$ is an arbitrary constant.

At the $\zeta=-\infty$ steady state the stability matrix becomes

$$
J_{\left(\tilde{U}_{0}, \tilde{P}_{0}\right)}=\left(\begin{array}{cc}
0 & \frac{a}{\hat{c}_{0} d_{u}} \\
-\frac{\hat{c}_{0}}{a} & \frac{1}{a}
\end{array}\right) .
$$

This matrix has eigenvalues

$$
\lambda=\frac{1 \pm \sqrt{1-4 a^{2} / d_{u}}}{2 a} .
$$

Thus the eigenvalues have a positive real part, and so this steady state is unstable and a smooth connection between the values at each end of the outer solution shock is possible. 


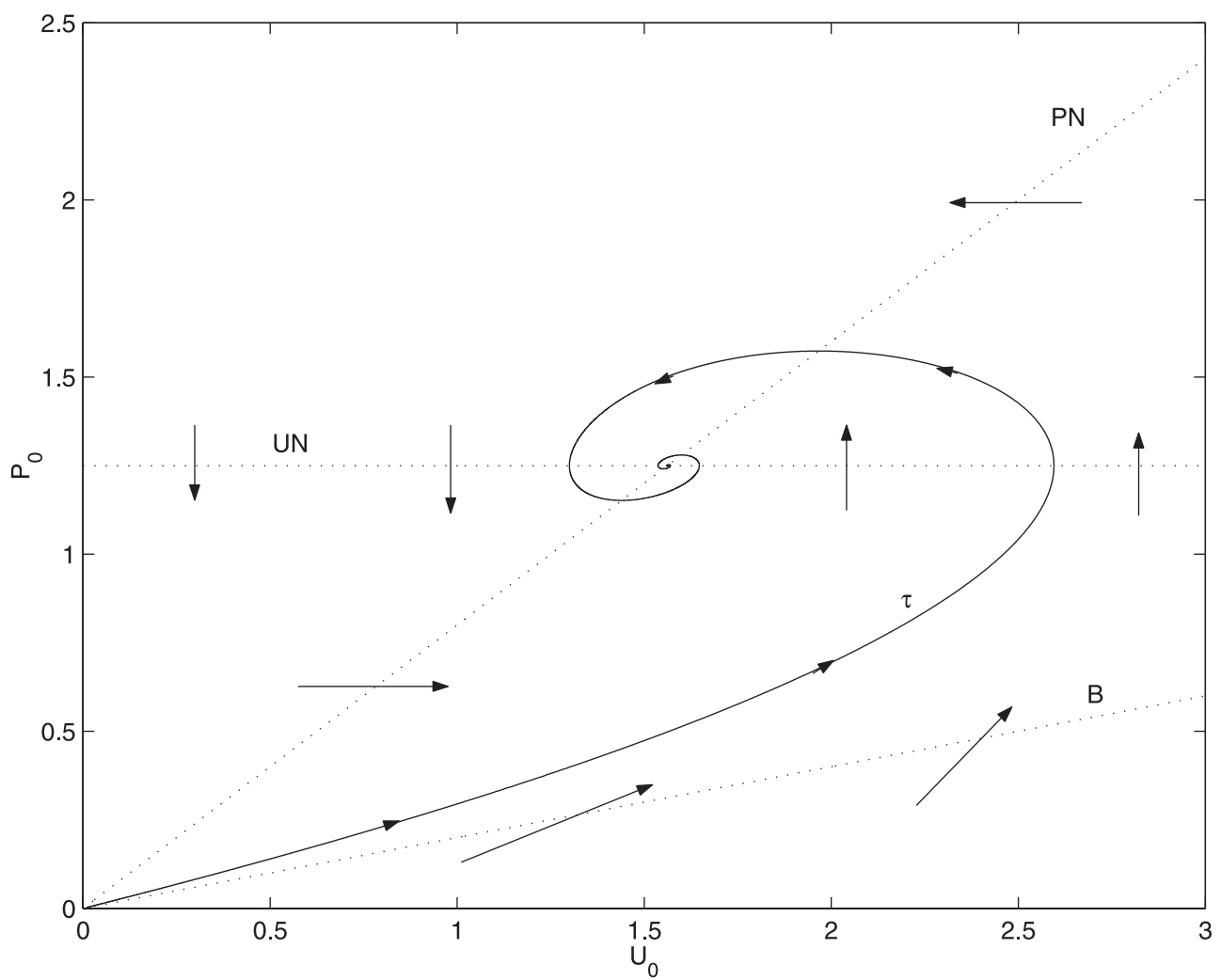

Figure 6. An illustration of the phase plane argument described in the text as to why a connection between the steady states in the $\left(\tilde{U}_{0}, \tilde{P}_{0}\right)$ phase plane must exist. The trajectory $\tau$ leaves the origin along the unstable manifold and enters the region bounded by the $\tilde{U}_{0}$ nullcline (labelled 'UN') the $\tilde{P}_{0}$ nullcline (labelled 'PN') and the line $\tilde{P}_{0}=\mu \tilde{U}_{0}$ (labelled 'B') where $\mu<\hat{c}_{0} d_{u} /\left(d_{u}+a^{2}\right)$. Along the $\tilde{P}_{0}$ nullcline and the line $\tilde{P}_{0}=\mu \tilde{U}_{0}$ the vector field points into this region. Therefore, $\tau$ may only leave the region by crossing the $\tilde{U}_{0}$ nullcline. This occurs at a value of $\tilde{U}_{0}=U^{*}$ say. If $U^{*}=a^{2} / \hat{c}_{0}^{2}$ then the connection is complete. Otherwise, $U^{*}>a^{2} / \hat{c}_{0}^{2}$, and the phase plane structure causes $\tau$ to spiral to the steady state as shown above. The arrows indicate the direction of the vector field.

To confirm that such a connection exists it is necessary to look at the global structure of the phase plane. With this aim we rewrite (42) and (44) in terms of $\zeta^{*}=-\zeta / a$

$$
\begin{aligned}
& \frac{\mathrm{d} \tilde{U}_{0}}{\mathrm{~d} \zeta^{*}}=\frac{\tilde{U}_{0} \hat{c}_{0}}{d_{u}}\left(\frac{a^{2}}{\hat{c}_{0}}-\tilde{P}_{0}\right) \\
& \frac{\mathrm{d} \tilde{P}_{0}}{\mathrm{~d} \zeta^{*}}=\tilde{U}_{0} \hat{c}_{0}-\tilde{P}_{0} .
\end{aligned}
$$

The required connection now corresponds to a trajectory which leaves $\left(\tilde{U}_{0}=0, \tilde{P}_{0}=0\right)$ and terminates at $\left(\tilde{U}_{0}=a^{2} / \hat{c}_{0}^{2}, \tilde{P}_{0}=a^{2} / \hat{c}_{0}\right)$. We have already shown that the origin is a saddle point and therefore exactly one trajectory leaves it along the unstable (in the $\zeta^{*}$ coordinate system) manifold $\left(1, \hat{c}_{0} d_{u} /\left(d_{u}+a^{2}\right)\right)$. We wish to prove that this trajectory, $\tau$, terminates at $\left(\tilde{U}_{0}=a^{2} / \hat{c}_{0}^{2}, \tilde{P}_{0}=a^{2} / \hat{c}_{0}\right)$. We consider a line in the $\left(\tilde{U}_{0}, \tilde{P}_{0}\right)$ phase plane, $\tilde{P}_{0}=\mu \tilde{U}_{0}$ where $\mu<d_{u} /\left(d_{u}+a^{2}\right)$. Both $\mathrm{d} \tilde{U}_{0} / \mathrm{d} \zeta^{*}$ and $\mathrm{d} \tilde{P}_{0} / \mathrm{d} \zeta^{*}$ can be shown to be positive on this line in the region where $\tilde{P}_{0}<a^{2} / \hat{c}_{0}$. 


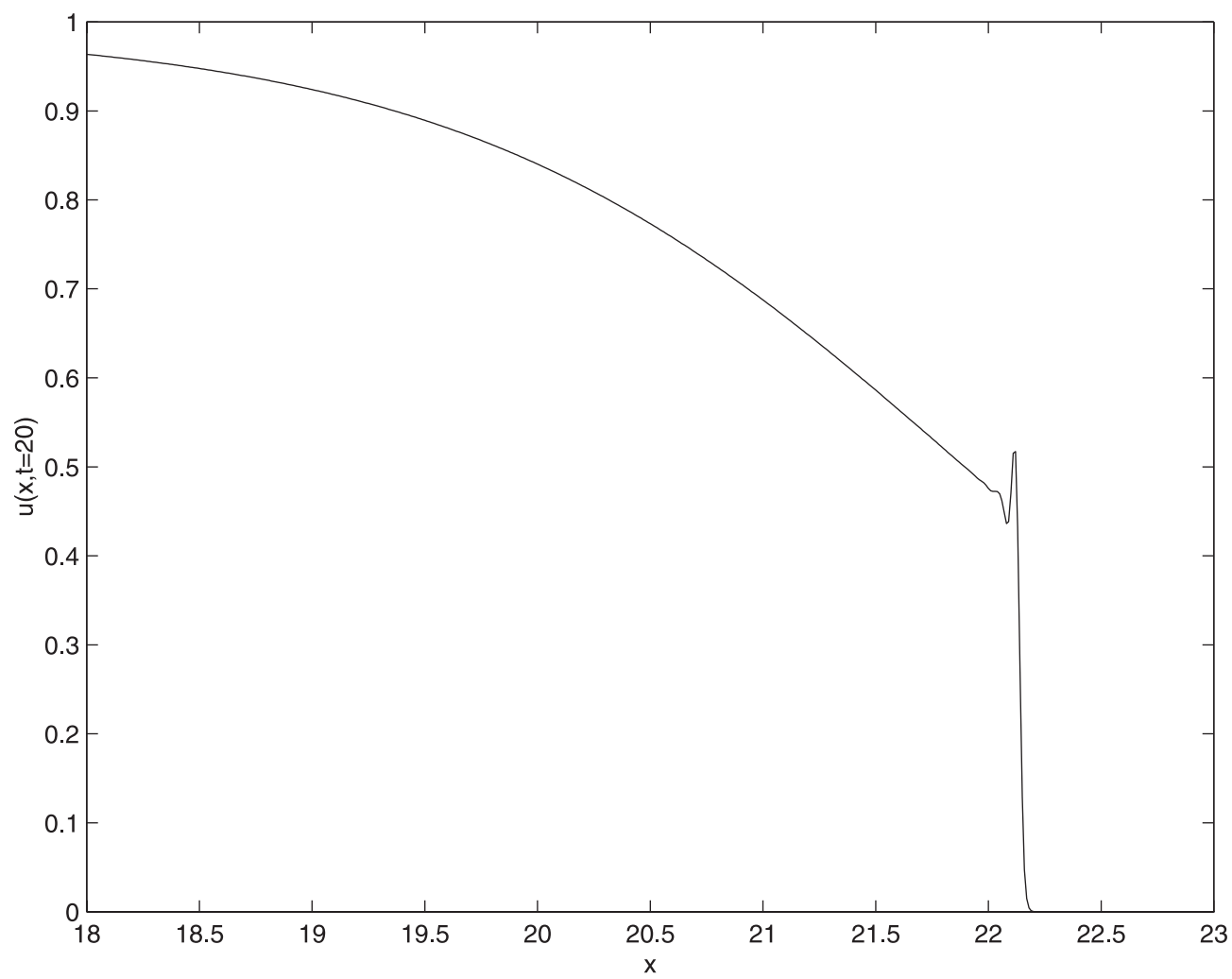

Figure 7. The $u(x, t=20)$ profile of a numerical travelling wave solution to (31)-(33) evolving from semi-compact initial conditions. The parameters are $\epsilon=0.01, \hat{c}=1.0$ and $D_{u}=0.005$ (i.e. $d_{u}=0.5$ ). The numerically measured wavespeed is $a=0.667$. The oscillation to the left of the smoothed shock can be clearly seen.

Referring to figure 6, upon leaving the origin, the trajectory $\tau$ enters the region which is bounded by the $\tilde{U}_{0}$ nullcline $\left(\tilde{P}_{0}=a^{2} / \hat{c}_{0}\right)$, the $\tilde{P}_{0}$ nullcline $\left(\tilde{P}_{0}=\hat{c}_{0} \tilde{U}_{0}\right)$ and the line $\tilde{P}_{0}=\mu \tilde{U}_{0}$. The trajectory must stay in this region until it reaches $\tilde{P}_{0}=a^{2} / \hat{c}_{0}$, at $\tilde{U}_{0}=U^{*}$ say. If $U^{*}=a^{2} / \hat{c}_{0}^{2}$ then the connection has been completed. Otherwise, $U^{*}>a^{2} / \hat{c}_{0}^{2}$, and the form of the phase plane means that $\tau$ must spiral round the steady state, passing through the half-lines $\tilde{P}_{0}=\hat{c}_{0} \tilde{U}_{0}\left(\tilde{P}_{0}>a^{2} / \hat{c}_{0}\right), \tilde{P}_{0}=a^{2} / \hat{c}_{0}\left(\tilde{U}_{0}<a^{2} / \hat{c}_{0}^{2}\right)$ and $\tilde{P}_{0}=\hat{c}_{0} \tilde{U}_{0}$ $\left(\tilde{P}_{0}>a^{2} / \hat{c}_{0}\right)$ in that order, thus re-entering the original region. Then $\tilde{U}_{0}$ and $\tilde{P}_{0}$ must increase along $\tau$ until $\tilde{P}_{0}=a^{2} / \hat{c}_{0}$ again. Since $\tau$ cannot cross itself, this must occur at a $\tilde{U}_{0}$ value less than $U^{*}$. By repeating this argument we can show that $\tau$ eventually spirals in to $\left(\tilde{U}_{0}=a^{2} / \hat{c}_{0}^{2}, \tilde{P}_{0}=a^{2} / \hat{c}_{0}\right)$.

Returning to $\zeta$ coordinates we have shown that the values of $\tilde{U}_{0}, \tilde{C}_{0}$ and $\tilde{P}_{0}$ at $\zeta= \pm \infty$ match with the values of $U_{0}, C_{0}$ and $P_{0}$ on either side of the outer solution shock. Furthermore, the behaviour close to the $\zeta=-\infty$ invading front steady state can also explain the oscillatory behaviour seen in the $u$ profile in figure 5, since when $d_{u}<4 a^{2}$ the eigenvalues (51) are complex and the steady state $\left(\tilde{U}_{0}=a^{2} / \hat{c}_{0}^{2}, \tilde{P}_{0}=a^{2} / \hat{c}_{0}\right)$ is a spiral. The amplitude of these oscillations increases as $d_{u} \rightarrow 0$ and if $d_{u}=0$ the eigenvectors become singular and no stable travelling wave solution exists. For $d_{u}>4 a^{2}$ the steady state is an unstable node and there are no oscillations in the $u$ and $p$ profiles, and this would be expected for a diffusion-dominated 


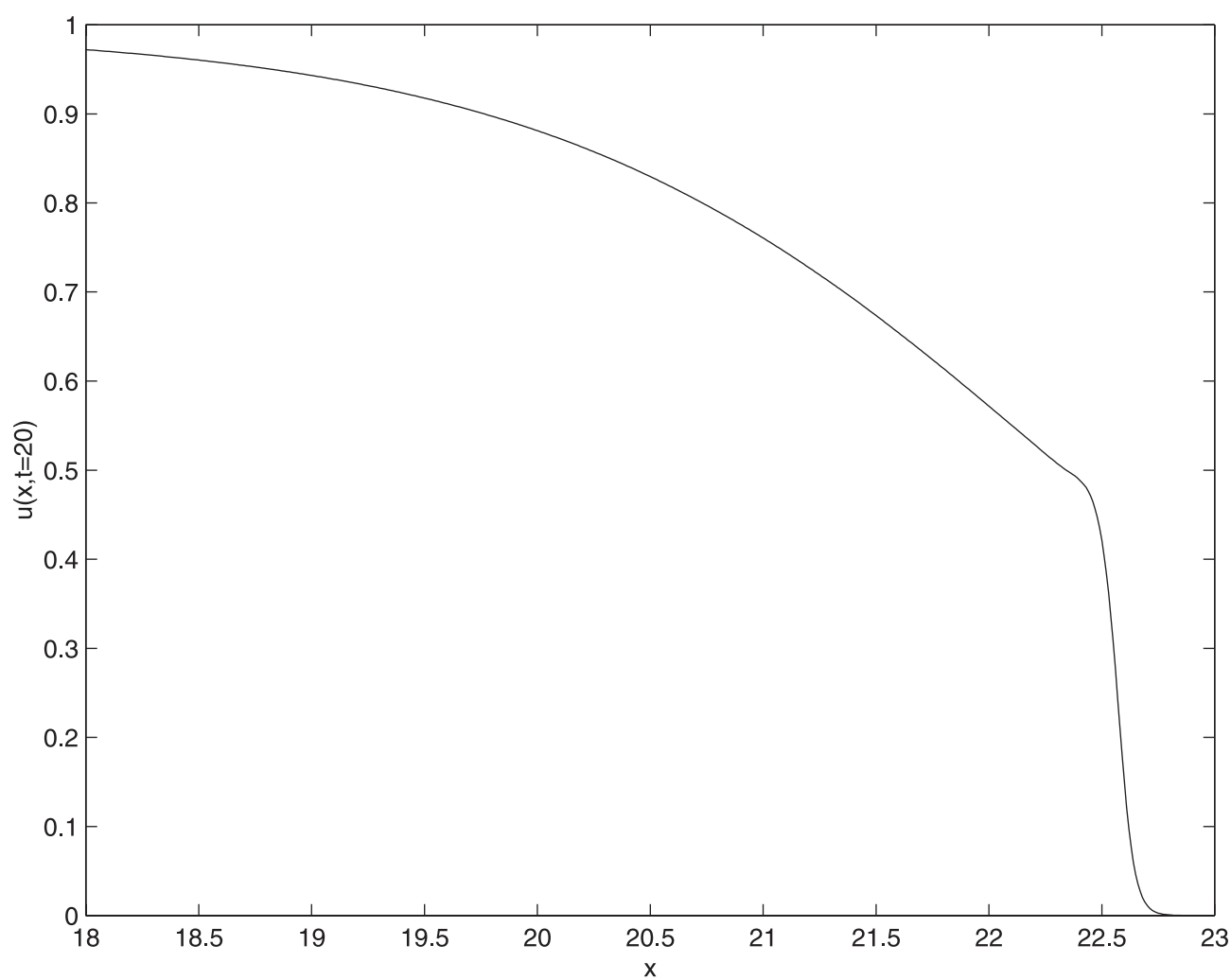

Figure 8. The $u(x, t=20)$ profile of a numerical travelling wave solution to (31)-(33) evolving from semi-compact initial conditions. The parameters are $\epsilon=0.01, \hat{c}=1.0$ and $D_{u}=0.02$ (i.e. $d_{u}=2.0$ ). The increased diffusion has caused the wavespeed to rise to $a=0.690$ and the oscillation to the left of the smoothed shock is no longer present.

process. We have thus shown that the blunt interface solution described in section 2 , forms a leading-order outer solution to a valid travelling wave solution of the full model with diffusion (31)-(33).

If we consider a specific example, we found in [7] that when $\hat{c}_{0}=1$, the wavespeed, $a_{0}$, of the blunt interface solution $\approx 0.66$ and thus the critical value of the rescaled diffusion coefficient, $d_{u} \approx 1.76$ or $D_{u} \approx 1.76 \epsilon$. In figure 7 we confirm that oscillations are present at the front of the $u(x, t)$ profile for $d_{u}$ significantly less than 1.76. In figure 8 we see that these oscillations are no longer present for $d_{u}=2.0$. The exact value of $d_{u}$ at which the oscillations disappear is difficult to assess from numerical solutions, but upon magnifying the solution there is some evidence of their presence when $d_{u}=1.4$. Both of these profiles bear a strong resemblance to the blunt interface solutions (figure 1) except in the rescaled inner layer. These numerical solutions are calculated by adding an explicit term to represent the diffusion to the Kurganov-Tadmor [5] scheme used in section 4.

As $d_{u}$ and $\epsilon$ decrease, the wavespeed of the travelling wave solution which evolves from semi-compact initial data approaches the wavespeed of the blunt solution to (5) and (6) as illustrated in figure 3. Here, for relatively large $\hat{c}$, the wavespeed of the three-variable model closely follows the approximation derived in [7]. For smaller values of $\hat{c}$ however, the haptotactic flux $u \mathrm{~d} c / \mathrm{d} x$ is smaller and diffusion dominates haptotaxis. Therefore, as $\hat{c}$ is 
reduced the wavespeed decays towards $2 \sqrt{D_{u}}$, the wavespeed of the minimum speed equation to the Fisher equation and other diffusion-dominated processes.

\section{Conclusions}

In this paper we have studied a three-equation model of malignant invasion, which initially neglected diffusion in order to focus upon the effect of haptotaxis in the process of cells invading surrounding tissue by producing degradative enzymes. We found, however, that a small amount of diffusion in the model has a vital stabilizing effect on the blunt invasion profiles that we expect to be of most physical significance. Aznavoorian et al [1] have demonstrated experimentally the existence of haptoxis-dominated motion of particular tumour lines such as HT1080 melanoma cells.

In previous work [7] we approximated this three-equation model without diffusion (2)(4) by a two-equation model (5) and (6), and found that novel discontinuous travelling wave solutions evolved from semi-compact initial data. At the front of these waves was a blunt interface or shock which took the concentration of invading cells from a positive value down to zero. In contrast to the conventional invading tails from diffusion-dominated equations which decay exponentially [3], these profiles have no cell density ahead of the blunt invasion face.

In the full model, when the diffusion coefficients and the parameter $\epsilon$ are small, these blunt solutions were a good approximation to the solution profiles of the full model with added diffusion (31)-(33), except for local detail at the blunt interface. At the invasion crest the behaviour of the solution profile was highly dependent upon the amount of diffusion, with a spike and oscillations growing as the diffusion is reduced. The wavespeed of the blunt solutions to the reduced model could be estimated from behaviour in a two-dimensional phase plane, and provided an accurate lower bound on the wavespeeds evolving from (31)-(33) for small diffusion and fast protease adjustment.

This work illustrates that the solutions to the reduced model are more than just a mathematical curiosity, since they do provide important information about the higher-order and more physically useful system (31)-(33). This work may also be relevant to other systems where the dominant motility mechanism is an advective (hyperbolic-like) term rather than diffusion.

The surprise here is that the solutions can stably evolve and steadily invade even though the solution profiles are constructed from ordinary differential equations that have singular behaviour zones in several regions of the solution profiles. These near singular solutions (for small diffusion and fast protease adjustment) have the slowest invasion speeds and the most interesting 'near-shock' profiles.

\section{Acknowledgments}

BPM was supported in part by a graduate studentship from EPSRC. JAS was supported by Research Development grant 107 from SHEFC and by an Advanced Research Fellowship from EPSRC.

\section{References}

[1] Aznavoorian S, Stracke M L, Krutzsch H, Schiffman E and Liotta L A 1990 Signal transduction for chemotaxis and haptotaxis by matrix molecules in tumour cells J. Cell Biol. 110 1427-38

[2] Courant R and Hilbert D 1962 Methods of Mathematical Physics II (New York: Interscience)

[3] Fisher R A 1937 The wave of advance of advantageous genes Ann. Eugenics 7 353-69 
[4] Kolmogorov A, Petrovsky A and Piscounoff N 1988 Study of the diffusion equation with growth of the quantity of matter and its application to a biology problem Dynamics of Curved Fronts (New York: Academic)

[5] Kurganov A and Tadmor E 1999 New high-resolution central schemes for nonlinear conservation laws and convection-diffusion equations Technical Report CAM 99-16 UCLA Computational and Applied Mathematics

[6] Leveque R J 1992 Numerical Methods for Conservation Laws (Boston, MA: Birkhäuser) 2nd edn

[7] Marchant B P, Norbury J and Perumpanani A J 2000 Travelling shock waves arising in a model of malignant invasion Siam J. Appl. Math. 60 463-76

[8] Murray J D 1989 Mathematical Biology (Berlin: Springer)

[9] Perumpanani A J, Norbury J, Sherratt J A and Byrne H M 1996 Biological inferences from a mathematical model for malignant invasion Invasion Metastasis 16 209-21

[10] Perumpanani A J, Norbury J, Sherratt J A and Byrne H M 1999 A two parameter family of travelling waves with a singular barrier arising from the modelling of matrix mediated malignant invasion Physica D 126 145-59

[11] Pettet G J, McElwain D L and Norbury J 2000 Lotka-Volterra equations with chemotaxis: walls, barriers and travelling waves Math. Appl. Med. Biol. 17 395-413

[12] Tsuboi R and Rifkin D B 1990 Bimodal relationship between the invasion of the amniotic membrane and plasminogen activator activity Int. J. Cancer 46 56-60 